\title{
ON SANDWICH THEOREMS FOR SOME CLASSES OF ANALYTIC FUNCTIONS
}

\author{
T. N. SHANMUGAM, S. SIVASUBRAMANIAN, AND H. SILVERMAN \\ Received 20 April 2006; Revised 31 July 2006; Accepted 5 September 2006
}

The purpose of this present paper is to derive some subordination and superordination results for certain normalized analytic functions in the open unit disk. Relevant connections of the results, which are presented in the paper, with various known results are also considered.

Copyright (c) 2006 Hindawi Publishing Corporation. All rights reserved.

\section{Introduction}

Let $\mathscr{H}$ be the class of functions analytic in $\Delta:=\{z:|z|<1\}$, and $\mathscr{H}[a, n]$ be the subclass of $\mathscr{H}$ consisting of functions of the form $f(z)=a+a_{n} z^{n}+a_{n+1} z^{n+1}+\cdots$. Let $\mathscr{A}$ be the subclass of $\mathcal{H}$ consisting of functions of the form $f(z)=z+a_{2} z^{2}+\cdots$. With a view to recalling the principle of subordination between analytic functions, let the functions $f$ and $g$ be analytic in $\Delta$. Then we say that the function $f$ is subordinate to $g$ if there exists a Schwarz function $\omega(z)$, analytic in $\Delta$ with

$$
\omega(0)=0, \quad|\omega(z)|<1 \quad(z \in \Delta),
$$

such that

$$
f(z)=g(\omega(z)) \quad(z \in \Delta)
$$

We denote this subordination by

$$
f \prec g \quad \text { or } \quad f(z) \prec g(z) \quad(z \in \Delta) \text {. }
$$

In particular, if the function $g$ is univalent in $\Delta$, the above subordination is equivalent to

$$
f(0)=g(0), \quad f(\Delta) \subset g(\Delta) .
$$

Hindawi Publishing Corporation International Journal of Mathematics and Mathematical Sciences Volume 2006, Article ID 29684, Pages 1-13

DOI 10.1155/IJMMS/2006/29684 
Let $p, h \in \mathcal{H}$ and let $\phi(r, s, t ; z): \mathbb{C}^{3} \times \Delta \rightarrow \mathbb{C}$. If $p$ and $\phi\left(p(z), z p^{\prime}(z), z^{2} p^{\prime \prime}(z) ; z\right)$ are univalent and if $p$ satisfies the second-order superordination

$$
h(z) \prec \phi\left(p(z), z p^{\prime}(z), z^{2} p^{\prime \prime}(z) ; z\right),
$$

then $p$ is a solution of the differential superordination (1.5). (If $f$ is subordinate to $F$, then $F$ is superordinate to $f$.) An analytic function $q$ is called a subordinant if $q \prec p$ for all $p$ satisfying (1.5). A univalent subordinant $\tilde{q}$ that satisfies $q \prec \tilde{q}$ for all subordinants $q$ of (1.5) is said to be the best subordinant. Recently Miller and Mocanu [5] obtained conditions on $h, q$, and $\phi$ for which the following implication holds:

$$
h(z) \prec \phi\left(p(z), z p^{\prime}(z), z^{2} p^{\prime \prime}(z) ; z\right) \Longrightarrow q(z) \prec p(z) .
$$

Using the results of Miller and Mocanu [5], Bulboacă [3] considered certain classes of first-order differential superordinations as well as superordination-preserving integral operators [2]. Ali et al. [1] have used the results of Bulboacă [3] and obtained sufficient conditions for certain normalized analytic functions $f(z)$ to satisfy

$$
q_{1}(z) \prec \frac{z f^{\prime}(z)}{f(z)} \prec q_{2}(z),
$$

where $q_{1}$ and $q_{2}$ are given univalent functions in $\Delta$ with $q_{1}(0)=1$ and $q_{2}(0)=1$. Shanmugam et al. [8] obtained sufficient conditions for normalized analytic functions $f(z)$ to satisfy

$$
\begin{gathered}
q_{1}(z) \prec \frac{f(z)}{z f^{\prime}(z)} \prec q_{2}(z), \\
q_{1}(z) \prec \frac{z^{2} f^{\prime}(z)}{\{f(z)\}^{2}} \prec q_{2}(z),
\end{gathered}
$$

where $q_{1}$ and $q_{2}$ are given univalent functions in $\Delta$ with $q_{1}(0)=1$ and $q_{2}(0)=1$, while Obradović and Owa [7] obtained subordination results with the quantity $(f(z) / z)^{\mu}$ (see also [10]).

Obradović [6] introduced a class of functions $f \in \mathscr{A}$ such that for $0<\alpha<1$,

$$
\Re\left\{f^{\prime}(z)\left(\frac{z}{f(z)}\right)^{\alpha}\right\}>0, \quad z \in \Delta .
$$

He called this class of function "non-Bazilevič" type. Tuneski and Darus [11] obtained Fekete-Szegö inequality for the non-Bazilevič class of functions. Using this non-Bazilevič class, Wang et al. [12] studied many subordination results for the class $N(\alpha, \lambda, A, B)$ defined as

$$
N(\alpha, \lambda, A, B):=\left\{f \in \mathscr{A}:(1+\lambda)\left(\frac{z}{f(z)}\right)^{\alpha}-\lambda f^{\prime}(z)\left(\frac{z}{f(z)}\right)^{1+\alpha} \prec \frac{1+A z}{1+B z}\right\},
$$

where $\lambda \in \mathbb{C},-1 \leq B \leq 1, A \neq B, 0<\alpha<1$. 
The main object of the present sequel to the aforementioned works is to apply a method based on the differential subordination in order to derive several subordination results. Furthermore, we obtain the previous results of Srivastava and Lashin [10], Singh [9] and Obradović and Owa [7] as special cases of some of the results presented here.

\section{Preliminaries}

In our present investigation, we will need the following definition and results.

Definition 2.1 (see [5, Definition 2, page 817]). Denote by $Q$ the set of all functions $f(z)$ that are analytic and injective on $\bar{\Delta}-E(f)$, where

$$
E(f)=\left\{\zeta \in \partial \Delta: \lim _{z \rightarrow \zeta} f(z)=\infty\right\}
$$

and are such that $f^{\prime}(\zeta) \neq 0$ for $\zeta \in \partial \Delta-E(f)$.

Theorem 2.2 (see [4, Theorem 3.4h, page 132]). Let $q(z)$ be univalent in the unit disk $\Delta$ and let $\theta$ and $\phi$ be analytic in a domain D containing $q(\Delta)$ with $\phi(w) \neq 0$ when $w \in q(\Delta)$. Set $Q(z)=z q^{\prime}(z) \phi(q(z)), h(z)=\theta(q(z))+Q(z)$. Suppose that

(1) $Q(z)$ is starlike univalent in $\Delta$;

(2) $\mathfrak{R}\left(z h^{\prime}(z)\right) / Q(z)>0$ for $z \in \Delta$.

If

$$
\theta(p(z))+z p^{\prime}(z) \phi(p(z)) \prec \theta(q(z))+z q^{\prime}(z) \phi(q(z))
$$

then $p(z) \prec q(z)$ and $q(z)$ is the best dominant.

Lemma 2.3 (see [8]). Let $q$ be a convex univalent function in $\Delta$ and let $\psi, \gamma \in \mathbb{C}$ with $\mathfrak{R}(1+$ $\left.\left(z q^{\prime \prime}(z) / q^{\prime}(z)\right)\right)>\max \{0,-\mathfrak{R}(\psi / \gamma)\}$. If $p(z)$ is analytic in $\Delta$ and

$$
\psi p(z)+\gamma z p^{\prime}(z) \prec \psi q(z)+\gamma z q^{\prime}(z)
$$

then $p(z) \prec q(z)$ and $q$ is the best dominant.

Lemma 2.4 (see [4, Corollary 3.4h.1, page 135]). Let $q(z)$ be univalent in $\Delta$ and let $\varphi(z)$ be analytic in a domain containing $q(\Delta)$. If $z q^{\prime}(z) / \varphi(q(z))$ is starlike, and

$$
z p^{\prime}(z) \varphi(p(z)) \prec z q^{\prime}(z) \varphi(q(z))
$$

then $p(z) \prec q(z)$ and $q$ is the best dominant.

Theorem 2.5 (see [3]). Let $q(z)$ be convex univalent in the unit disk $\Delta$ and let $\vartheta$ and $\varphi$ be analytic in a domain $D$ containing $q(\Delta)$. Suppose that

(1) $\mathfrak{R}\left[\vartheta^{\prime}(q(z)) / \varphi(q(z))\right]>0$ for $z \in \Delta$;

(2) $z q^{\prime}(z) \varphi(q(z))$ is starlike univalent in $\Delta$. 
If $p(z) \in \mathscr{H}[q(0), 1] \cap Q$, with $p(\Delta) \subseteq D$, and $\vartheta(p(z))+z p^{\prime}(z) \varphi(p(z))$ is univalent in $\Delta$, and

$$
\vartheta(q(z))+z q^{\prime}(z) \varphi(q(z)) \prec \vartheta(p(z))+z p^{\prime}(z) \varphi(p(z)),
$$

then $q(z) \prec p(z)$ and $q$ is the best subordinant.

Lemma 2.6 (see [5, Theorem 8, page 822]). Let $q$ be convex univalent in $\Delta$ and $\gamma \in \mathbb{C}$. Further assume that $\mathfrak{R}[\bar{\gamma}]>0$. If $p(z) \in \mathscr{H}[q(0), 1] \cap Q$, and $p(z)+\gamma z p^{\prime}(z)$ is univalent in $\Delta$, then

$$
q(z)+\gamma z q^{\prime}(z) \prec p(z)+\gamma z p^{\prime}(z)
$$

implies $q(z) \prec p(z)$ and $q$ is the best subordinant.

\section{Subordination for analytic functions}

By using Lemma 2.3, we first prove the following.

Theorem 3.1. Let $q$ be univalent in $\Delta, \lambda \in \mathbb{C}$, and $0<\alpha<1$. Suppose $q$ satisfies

$$
\mathfrak{R}\left(1+\frac{z q^{\prime \prime}(z)}{q^{\prime}(z)}\right)>\max \left\{0,-\mathfrak{R}\left\{\frac{\lambda}{\alpha}\right\}\right\} .
$$

If $f \in \mathscr{A}$ satisfies the subordination

$$
(1+\lambda)\left(\frac{z}{f(z)}\right)^{\alpha}-\lambda f^{\prime}(z)\left(\frac{z}{f(z)}\right)^{1+\alpha} \prec q(z)+\frac{\lambda z q^{\prime}(z)}{\alpha},
$$

then

$$
\left(\frac{z}{f(z)}\right)^{\alpha} \prec q(z)
$$

and $q$ is the best dominant.

Proof. Define the function $p(z)$ by

$$
p(z):=\left(\frac{z}{f(z)}\right)^{\alpha} .
$$

Then

$$
\frac{z p^{\prime}(z)}{p(z)}=\alpha\left[1-\frac{z f^{\prime}(z)}{f(z)}\right]
$$

which, in light of hypothesis (3.2) of Theorem 3.1, yields the following subordination:

$$
p(z)+\frac{\lambda z p^{\prime}(z)}{\alpha} \prec q(z)+\frac{\lambda z q^{\prime}(z)}{\alpha} .
$$

The assertion of Theorem 3.1 now follows by an application of Lemma 2.3 with $\gamma=\lambda / \alpha$ and $\psi=1$. 
Taking $q(z)=(1+A z) /(1+B z)$ in Theorem 3.1, we have the following corollary. Corollary 3.2. Let $-1 \leq B<A \leq 1$ and (3.1) hold. If $f \in \mathscr{A}$, and

$$
(1+\lambda)\left(\frac{z}{f(z)}\right)^{\alpha}-\lambda f^{\prime}(z)\left(\frac{z}{f(z)}\right)^{1+\alpha} \prec \frac{\lambda(A-B) z}{\alpha(1+B z)^{2}}+\frac{1+A z}{1+B z},
$$

then

$$
\left(\frac{z}{f(z)}\right)^{\alpha} \prec \frac{1+A z}{1+B z}
$$

and $(1+A z) /(1+B z)$ is the best dominant.

Theorem 3.1 for the choice of $q(z)=(1+z) /(1-z)$ reduces to the following. Corollary 3.3. Let (3.1) hold. If $f \in \mathscr{A}$, and

$$
(1+\lambda)\left(\frac{z}{f(z)}\right)^{\alpha}-\lambda f^{\prime}(z)\left(\frac{z}{f(z)}\right)^{1+\alpha} \prec \frac{2 \lambda z}{\alpha(1-z)^{2}}+\frac{1+z}{1-z},
$$

then

$$
\left(\frac{z}{f(z)}\right)^{\alpha} \prec \frac{1+z}{1-z}
$$

and $(1+z) /(1-z)$ is the best dominant.

Theorem 3.4. Let $q$ be univalent in $\Delta, \gamma, \mu \neq 0 \in \mathbb{C}$, and $0 \leq \beta \leq 1$. Let $f \in \mathscr{A}$. Suppose $q$ satisfies

$$
\mathfrak{R}\left\{1+\frac{z q^{\prime \prime}(z)}{q^{\prime}(z)}-\frac{z q^{\prime}(z)}{q(z)}\right\}>0
$$

If

$$
1+\gamma \mu\left\{\frac{z f^{\prime}(z)+\beta z^{2} f^{\prime \prime}(z)}{(1-\beta) f(z)+\beta z f^{\prime}(z)}-1\right\} \prec 1+\gamma \frac{z q^{\prime}(z)}{q(z)}
$$

then

$$
\left[\frac{(1-\beta) f(z)+\beta z f^{\prime}(z)}{z}\right]^{\mu} \prec q(z)
$$

and $q$ is the best dominant.

Proof. Define the function $p(z)$ by

$$
p(z):=\left[\frac{(1-\beta) f(z)+\beta z f^{\prime}(z)}{z}\right]^{\mu}, \quad z \neq 0
$$


Then a computation shows that

$$
\mu\left\{\frac{z f^{\prime}(z)+\beta z^{2} f^{\prime \prime}(z)}{(1-\beta) f(z)+\beta z f^{\prime}(z)}-1\right\}=\frac{z p^{\prime}(z)}{p(z)} .
$$

By setting

$$
\theta(\omega):=1, \quad \phi(\omega):=\frac{\gamma}{\omega},
$$

it can be easily observed that $\theta(\omega)$ is analytic in $\mathbb{C}, \phi(\omega)$ is analytic in $\mathbb{C} \backslash\{0\}$, and that

$$
\phi(\omega) \neq 0 \quad(\omega \in \mathbb{C} \backslash\{0\}) .
$$

Also, we let

$$
\begin{gathered}
Q(z)=z q^{\prime}(z) \phi(q(z))=\gamma \frac{z q^{\prime}(z)}{q(z)}, \\
h(z)=\theta\{q(z)\}+Q(z)=1+\gamma \frac{z q^{\prime}(z)}{q(z)} .
\end{gathered}
$$

From (3.11), we find that $Q(z)$ is starlike univalent in $\Delta$ and that

$$
\mathfrak{R}\left(\frac{z h^{\prime}(z)}{Q(z)}\right)=\mathfrak{R}\left\{1+\frac{z q^{\prime \prime}(z)}{q^{\prime}(z)}-\frac{z q^{\prime}(z)}{q(z)}\right\}>0
$$

by the hypothesis (3.11) of Theorem 3.4. Thus, by applying Theorem 2.2, our proof of Theorem 3.4 is completed.

For a special case when $q(z)=1 /(1-z)^{2 b}(b \in \mathbb{C} \backslash\{0\}), \beta=0, \gamma=1 / b$, and $\mu=1$, Theorem 3.4 reduces at once to the following known result obtained by Srivastava and Lashin [10].

Corollary 3.5. Let $b$ be a nonzero complex number. If $f \in \mathscr{A}$, and

$$
1+\frac{1}{b}\left[\frac{z f^{\prime}(z)}{f(z)}-1\right] \prec \frac{1+z}{1-z}
$$

then

$$
\frac{f(z)}{z} \prec \frac{1}{(1-z)^{2 b}}
$$

and $1 /(1-z)^{2 b}$ is the best dominant.

For a special case when $q(z)=1 /(1-z)^{2 b}(b \in \mathbb{C} \backslash\{0\}), \beta=1, \gamma=1 / b$, and $\mu=1$ Theorem 3.4 reduces at once to another known result obtained by Srivastava and Lashin [10]. 
T. N. Shanmugam et al. 7

Corollary 3.6. Let $b$ be a nonzero complex number. If $f \in \mathscr{A}$, and

$$
1+\frac{1}{b} \frac{z f^{\prime \prime}(z)}{f^{\prime}(z)} \prec \frac{1+z}{1-z},
$$

then

$$
f^{\prime}(z) \prec \frac{1}{(1-z)^{2 b}}
$$

and $1 /(1-z)^{2 b}$ is the best dominant.

For $q(z)=(1+B z)^{\mu(A-B) / B}, \gamma=1 / \mu$, and $\beta=0$ in Theorem 3.4, we get the following known result obtained by Obradović and Owa [7].

Corollary 3.7. Let $-1 \leq B<A \leq 1$. If $f \in \mathcal{A}$, and

$$
\frac{z f^{\prime}(z)}{f(z)} \prec \frac{1+A z}{1+B z}
$$

then

$$
\left(\frac{f(z)}{z}\right)^{\mu} \prec(1+B z)^{\mu(A-B) / B} \quad(z \in \Delta ; z \neq 0 ; \mu \in \mathbb{C} ; \mu \neq 0)
$$

and $(1+B z)^{\mu(A-B) / B}$ is the best dominant.

We remark here that $q(z)=(1+B z)^{\mu(A-B) / B}$ is univalent if and only if $\mid(\mu(A-B) / B)-$ $1 \mid \leq 1$ or $|(\mu(A-B) / B)+1| \leq 1$.

For $q(z)=e^{\mu A z}, \gamma=1 / \mu$, and $\beta=0$ in Theorem 3.4, we get the following known result obtained by Obradović and Owa [7].

Corollary 3.8. If $f \in \mathcal{A}$, and

$$
\frac{z f^{\prime}(z)}{f(z)} \prec 1+A z
$$

then

$$
\left(\frac{f(z)}{z}\right)^{\mu} \prec e^{\mu A z} \quad(z \in \Delta ; z \neq 0 ; \mu \in \mathbb{C} ; \mu \neq 0)
$$

and $e^{\mu A z}$ is the best dominant.

Similar to the previous corollary, the function $q(z)=e^{\mu A z}$ is univalent if and only if $|\mu A|<\pi$.

Theorem 3.9. Let $q$ be univalent in $\Delta, \gamma \neq 0, \delta, \alpha \in \mathbb{C}$, and let $0 \leq \beta \leq 1$. Let $f \in A$. Suppose q satisfies

$$
\mathfrak{R}\left\{\frac{\alpha}{\gamma}+1+\frac{z q^{\prime \prime}(z)}{q^{\prime}(z)}\right\}>0
$$


and also $\mathfrak{R}(\alpha / \gamma)>0$. Let

$$
\Psi(z):=\left[\frac{(1-\beta) f(z)+\beta z f^{\prime}(z)}{z}\right]^{\mu}\left\{\alpha+\gamma \mu\left\{\frac{z f^{\prime}(z)+\beta z^{2} f^{\prime \prime}(z)}{(1-\beta) f(z)+\beta z f^{\prime}(z)}-1\right\}\right\}+\delta .
$$

If

$$
\Psi(z) \prec \alpha q(z)+\delta+\gamma z q^{\prime}(z),
$$

then

$$
\left[\frac{(1-\beta) f(z)+\beta z f^{\prime}(z)}{z}\right]^{\mu} \prec q(z)
$$

and $q$ is the best dominant.

Proof. Define the function $p(z)$ by

$$
p(z):=\left[\frac{(1-\beta) f(z)+\beta z f^{\prime}(z)}{z}\right]^{\mu} .
$$

Then a computation shows that

$$
\mu\left\{\frac{z f^{\prime}(z)+\beta z^{2} f^{\prime \prime}(z)}{(1-\beta) f(z)+\beta z f^{\prime}(z)}-1\right\}=\frac{z p^{\prime}(z)}{p(z)}
$$

and hence

$$
\mu p(z)\left\{\frac{z f^{\prime}(z)+\beta z^{2} f^{\prime \prime}(z)}{(1-\beta) f(z)+\beta z f^{\prime}(z)}-1\right\}=z p^{\prime}(z)
$$

By setting

$$
\theta(\omega):=\alpha \omega+\delta, \quad \phi(\omega):=\gamma,
$$

it can be easily observed that $\theta(\omega)$ and $\phi(\omega)$ are analytic in $\mathbb{C}$. Also, we let

$$
\begin{gathered}
Q(z)=z q^{\prime}(z) \phi(q(z))=\gamma z q^{\prime}(z), \\
h(z)=\theta\{q(z)\}+Q(z)=\alpha q(z)+\delta+\gamma z q^{\prime}(z) .
\end{gathered}
$$

From (3.28), we find that $Q(z)$ is starlike univalent in $\Delta$, and that

$$
\mathfrak{R}\left(\frac{z h^{\prime}(z)}{Q(z)}\right)=\mathfrak{R}\left\{\frac{\alpha}{\gamma}+1+\frac{z q^{\prime \prime}(z)}{q^{\prime}(z)}\right\}>0
$$

by the hypothesis (3.28) of Theorem 3.9. Thus, by applying Theorem 2.2, our proof of Theorem 3.9 is completed. 
T. N. Shanmugam et al. 9

For $\beta=1, \delta=-\alpha, \gamma=1$, we get the following corollary.

Corollary 3.10. Let $q$ be univalent in $\Delta$. Let $f \in \mathscr{A}$ and $1+\alpha>0$. Suppose $f$ satisfies

$$
\mathfrak{R}\left\{\alpha+1+\frac{z q^{\prime \prime}(z)}{q^{\prime}(z)}\right\}>0 .
$$

If

$$
\alpha\left\{\left(f^{\prime}(z)\right)^{\mu}-1\right\}+\mu\left\{\frac{z f^{\prime \prime}(z)}{f^{\prime}(z)}\left(f^{\prime}(z)\right)^{\mu}\right\} \prec \alpha q(z)-\alpha+z q^{\prime}(z)
$$

then

$$
\left[f^{\prime}(z)\right]^{\mu} \prec q(z)
$$

and $q$ is the best dominant.

Taking $q(z)=1+\lambda /(1+\alpha) z$, we obtain a recent result of Singh [9, Theorem 1 (ii), page 571].

\section{Superordination for analytic functions}

Theorem 4.1. Let $q$ be convex univalent in $\Delta, \lambda \in \mathbb{C}$, and $0<\alpha<1$. Suppose q satisfies

$$
\Re\{\lambda\}>0
$$

and $(z / f(z))^{\alpha} \in \mathscr{H}[q(0), 1] \cap$ Q. Let

$$
(1+\lambda)\left(\frac{z}{f(z)}\right)^{\alpha}-\lambda f^{\prime}(z)\left(\frac{z}{f(z)}\right)^{1+\alpha}
$$

be univalent in $\Delta$. If

$$
q(z)+\frac{\lambda z q^{\prime}(z)}{\alpha} \prec(1+\lambda)\left(\frac{z}{f(z)}\right)^{\alpha}-\lambda f^{\prime}(z)\left(\frac{z}{f(z)}\right)^{1+\alpha},
$$

then

$$
q(z) \prec\left(\frac{z}{f(z)}\right)^{\alpha}
$$

and $q$ is the best subordinant.

Proof. Define the function $p(z)$ by

$$
p(z):=\left(\frac{z}{f(z)}\right)^{\alpha} .
$$


Then a computation shows that

$$
p(z)+\frac{\lambda}{\alpha} z p^{\prime}(z)=(1+\lambda)\left(\frac{z}{f(z)}\right)^{\alpha}-\lambda f^{\prime}(z)\left(\frac{z}{f(z)}\right)^{1+\alpha} .
$$

Theorem 4.1 follows as an application of Lemma 2.6.

Taking $q(z)=(1+A z) /(1+B z)$ in Theorem 4.1, we get the following corollary.

Corollary 4.2. Let $-1 \leq B<A \leq 1$. Let $q$ be convex univalent in $\Delta$. Suppose q satisfies $\mathfrak{R}(\lambda)>0$ and $(z / f(z))^{\alpha} \in \mathscr{H}[q(0), 1] \cap Q$. Let

$$
(1+\lambda)\left(\frac{z}{f(z)}\right)^{\alpha}-\lambda f^{\prime}(z)\left(\frac{z}{f(z)}\right)^{1+\alpha}
$$

be univalent in $\Delta$. If

$$
\frac{\lambda(A-B) z}{\alpha(1+B z)^{2}}+\frac{1+A z}{1+B z} \prec(1+\lambda)\left(\frac{z}{f(z)}\right)^{\alpha}-\lambda f^{\prime}(z)\left(\frac{z}{f(z)}\right)^{1+\alpha},
$$

then

$$
\frac{1+A z}{1+B z} \prec\left(\frac{z}{f(z)}\right)^{\alpha}
$$

and $(1+A z) /(1+B z)$ is the best subordinant.

Since the proof of Theorem 5.2 is similar to the proof of Theorem 4.1, we state the theorem without proof.

Theorem 4.3. Let $q$ be convex univalent in $\Delta, \gamma \in \mathbb{C}, 0 \leq \beta \leq 1$, and $f \in \mathscr{A}$. Suppose $[((1-$ $\left.\left.\beta) f(z)+\beta z f^{\prime}(z)\right) / z\right]^{\mu} \in \mathscr{H}[q(0), 1] \cap Q$, and

$$
1+\gamma \mu\left\{\frac{z f^{\prime}(z)+\beta z^{2} f^{\prime \prime}(z)}{(1-\beta) f(z)+\beta z f^{\prime}(z)}-1\right\}
$$

is univalent in $\Delta$. If

$$
1+\gamma \frac{z q^{\prime}(z)}{q(z)} \prec 1+\gamma \mu\left\{\frac{z f^{\prime}(z)+\beta z^{2} f^{\prime \prime}(z)}{(1-\beta) f(z)+\beta z f^{\prime}(z)}-1\right\},
$$

then

$$
q(z) \prec\left[\frac{(1-\beta) f(z)+\beta z f^{\prime}(z)}{z}\right]^{\mu}
$$

and $q$ is the best subordinant.

Theorem 4.4. Let $q$ be convex univalent in $\Delta, \gamma \neq 0, \delta, \alpha \in \mathbb{C}$, and let $0 \leq \beta \leq 1$. Let $f \in \mathscr{A}$. Suppose q satisfies

$$
\mathfrak{R}\left\{\frac{\alpha}{\gamma} q^{\prime}(z)\right\}>0
$$


If

$\alpha q(z)+\delta+\gamma z q^{\prime}(z) \prec\left[\frac{(1-\beta) f(z)+\beta z f^{\prime}(z)}{z}\right]^{\mu}\left\{\alpha+\gamma \mu\left\{\frac{z f^{\prime}(z)+\beta z^{2} f^{\prime \prime}(z)}{(1-\beta) f(z)+\beta z f^{\prime}(z)}-1\right\}\right\}+\delta$,

then

$$
q(z) \prec\left[\frac{(1-\beta) f(z)+\beta z f^{\prime}(z)}{z}\right]^{\mu}
$$

and $q$ is the best subordinant.

Proof. Define the function $p(z)$ by

$$
p(z):=\left[\frac{(1-\beta) f(z)+\beta z f^{\prime}(z)}{z}\right]^{\mu} .
$$

Then a computation shows that

$$
\mu\left\{\frac{z f^{\prime}(z)+\beta z^{2} f^{\prime \prime}(z)}{(1-\beta) f(z)+\beta z f^{\prime}(z)}-1\right\}=\frac{z p^{\prime}(z)}{p(z)},
$$

and hence

$$
\mu p(z)\left\{\frac{z f^{\prime}(z)+\beta z^{2} f^{\prime \prime}(z)}{(1-\beta) f(z)+\beta z f^{\prime}(z)}-1\right\}=z p^{\prime}(z) .
$$

By setting

$$
\vartheta(\omega):=\alpha \omega+\delta, \quad \phi(\omega):=\gamma
$$

it can be easily observed that both $\theta(\omega)$ and $\phi(\omega)$ are analytic in $\mathbb{C}$. Now,

$$
\mathfrak{R}\left(\frac{\vartheta^{\prime}(q(z))}{\varphi(q(z))}\right)=\mathfrak{R}\left\{\frac{\alpha q^{\prime}(z)}{\gamma}\right\}>0
$$

by the hypothesis (4.13) of Theorem 4.4. Thus, by applying Theorem 2.5, our proof of Theorem 4.4 is completed.

\section{Sandwich results}

Combining the results of differential subordination and superordination, we state the following "sandwich results."

Theorem 5.1. Let $q_{1}$ be convex univalent and let $q_{2}$ be univalent in $\Delta, \lambda \in \mathbb{C}$, and $0<$ $\alpha<1$. Suppose $q_{1}$ satisfies (4.1) and $q_{2}$ satisfies (3.1). If $0 \neq(z / f(z))^{\alpha} \in \mathscr{H}[q(0), 1] \cap Q$, $(1+\lambda)(z / f(z))^{\alpha}-\lambda f^{\prime}(z)(z / f(z))^{1+\alpha}$ is univalent in $\Delta$, and

$$
q_{1}(z)+\frac{\lambda}{\alpha} z q_{1}^{\prime}(z) \prec(1+\lambda)\left(\frac{z}{f(z)}\right)^{\alpha}-\lambda f^{\prime}(z)\left(\frac{z}{f(z)}\right)^{1+\alpha} \prec q_{2}(z)+\frac{\lambda}{\alpha} z q_{2}^{\prime}(z),
$$


then

$$
q_{1}(z) \prec\left(\frac{z}{f(z)}\right)^{\alpha} \prec q_{2}(z)
$$

and $q_{1}$ and $q_{2}$ are, respectively, the best subordinant and best dominant.

Theorem 5.2. Let $q_{1}$ be convex univalent and let $q_{2}$ be univalent in $\Delta, \gamma \neq 0 \in \mathbb{C}, \mu \neq 0 \in \mathbb{C}$, $0 \leq \beta \leq 1$, and $q_{2}$ satisfies (3.11). Let $f \in \mathscr{A}$. Suppose $0 \neq\left[\left((1-\beta) f(z)+\beta z f^{\prime}(z) / z\right)\right]^{\mu} \in$ $\mathscr{H}[q(0), 1] \cap Q$,

$$
1+\gamma \mu\left\{\frac{z f^{\prime}(z)+\beta z^{2} f^{\prime \prime}(z)}{(1-\beta) f(z)+\beta z f^{\prime}(z)}-1\right\}
$$

is univalent in $\Delta$. If

$$
1+\gamma \frac{z q_{1}^{\prime}(z)}{q_{1}(z)} \prec 1+\gamma \mu\left\{\frac{z f^{\prime}(z)+\beta z^{2} f^{\prime \prime}(z)}{(1-\beta) f(z)+\beta z f^{\prime}(z)}-1\right\} \prec 1+\gamma \frac{z q_{2}^{\prime}(z)}{q_{2}(z)},
$$

then

$$
q_{1}(z) \prec\left[\frac{(1-\beta) f(z)+\beta z f^{\prime}(z)}{z}\right]^{\mu} \prec q_{2}(z)
$$

and $q_{1}$ and $q_{2}$ are, respectively, the best subordinant and the best dominant.

Theorem 5.3. Let $q_{1}$ be convex univalent and let $q_{2}$ be univalent in $\Delta, \gamma \neq 0 \in \mathbb{C}, \mu \neq$ $0 \in \mathbb{C}$ and $0 \leq \beta \leq 1$. Suppose $q_{1}$ satisfies (4.13), $q_{2}$ satisfies (3.28), and $[((1-\beta) f(z)+$ $\left.\left.\beta z f^{\prime}(z)\right) / z\right]^{\mu} \in \mathscr{H}[q(0), 1] \cap Q$. Let

$$
\left[\frac{(1-\beta) f(z)+\beta z f^{\prime}(z)}{z}\right]^{\mu}\left\{\alpha+\gamma \mu\left\{\frac{z f^{\prime}(z)+\beta z^{2} f^{\prime \prime}(z)}{(1-\beta) f(z)+\beta z f^{\prime}(z)}-1\right\}\right\}+\delta
$$

be univalent in $\Delta$. If

$$
\begin{aligned}
\alpha q_{1}(z) & +\delta+\gamma z q_{1}^{\prime}(z) \\
& \prec\left[\frac{(1-\beta) f(z)+\beta z f^{\prime}(z)}{z}\right]^{\mu}\left\{\alpha+\gamma \mu\left\{\frac{z f^{\prime}(z)+\beta z^{2} f^{\prime \prime}(z)}{(1-\beta) f(z)+\beta z f^{\prime}(z)}-1\right\}\right\}+\delta \\
& \prec \alpha q_{2}(z)+\delta+\gamma z q_{2}^{\prime}(z),
\end{aligned}
$$

then

$$
q_{1}(z) \prec\left[\frac{(1-\beta) f(z)+\beta z f^{\prime}(z)}{z}\right]^{\mu} \prec q_{2}(z)
$$

and $q_{1}$ and $q_{2}$ are, respectively, the best subordinant and the best dominant. 


\section{References}

[1] R. M. Ali, V. Ravichandran, M. H. Khan, and K. G. Subramanian, Differential sandwich theorems for certain analytic functions, Far East Journal of Mathematical Sciences 15 (2004), no. 1, 87-94.

[2] T. Bulboacă, A class of superordination-preserving integral operators, Indagationes Mathematicae. New Series 13 (2002), no. 3, 301-311.

[3] _ Classes of first-order differential superordinations, Demonstratio Mathematica 35 (2002), no. 2, 287-292.

[4] S. S. Miller and P. T. Mocanu, Differential Subordinations. Theory and Applications, Monographs and Textbooks in Pure and Applied Mathematics, vol. 225, Marcel Dekker, New York, 2000.

[5] ___ Subordinants of differential superordinations, Complex Variables 48 (2003), no. 10, 815826.

[6] M. Obradović, A class of univalent functions, Hokkaido Mathematical Journal 27 (1998), no. 2, 329-335.

[7] M. Obradović and S. Owa, On certain properties for some classes of starlike functions, Journal of Mathematical Analysis and Applications 145 (1990), no. 2, 357-364.

[8] T. N. Shanmugam, V. Ravichandran, and S. Sivasubramanian, Differential sandwich theorems for some subclasses of analytic functions, The Australian Journal of Mathematical Analysis and Applications 3 (2006), no. 1, 11, article 8.

[9] V. Singh, On some criteria for univalence and starlikeness, Indian Journal of Pure and Applied Mathematics 34 (2003), no. 4, 569-577.

[10] H. M. Srivastava and A. Y. Lashin, Some applications of the Briot-Bouquet differential subordination, Journal of Inequalities in Pure and Applied Mathematics 6 (2005), no. 2, 7, article 41.

[11] N. Tuneski and M. Darus, Fekete-Szegö functional for non-Bazilevič functions, Acta Mathematica. Academiae Paedagogicae Nyíregyháziensis. New Series 18 (2002), no. 2, 63-65.

[12] Z. Wang, C. Gao, and M. Liao, On certain generalized class of non-Bazilevič functions, Acta Mathematica. Academiae Paedagogicae Nyíregyháziensis. New Series 21 (2005), no. 2, 147-154.

T. N. Shanmugam: Department of Mathematics, College of Engineering, Anna University,

Chennai 600 025, India

E-mail address: shan@annauniv.edu

S. Sivasubramanian: Department of Mathematics, Easwari Engineering College, Ramapuram, Chennai 600 089, India

E-mail address: sivasaisastha@rediffmail.com

H. Silverman: College of Charleston, Charleston, SC 29424, USA

E-mail address: silvermanh@cofc.edu 


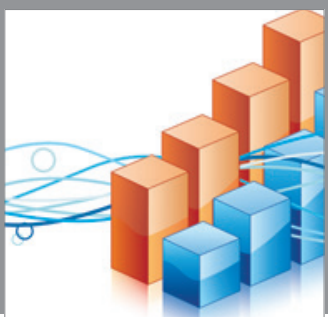

Advances in

Operations Research

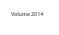

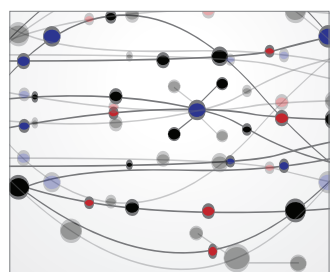

\section{The Scientific} World Journal
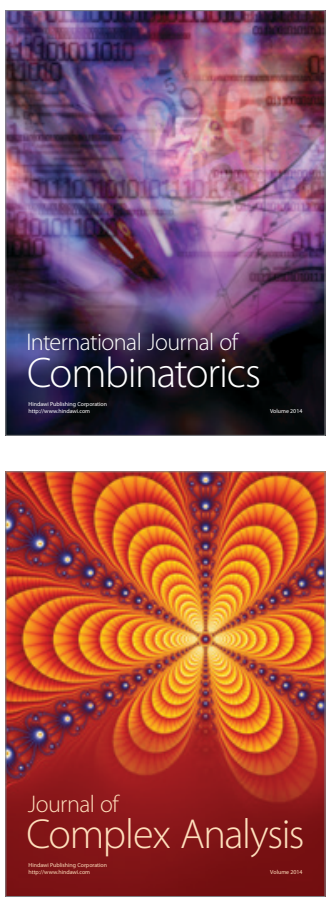

International Journal of

Mathematics and

Mathematical

Sciences
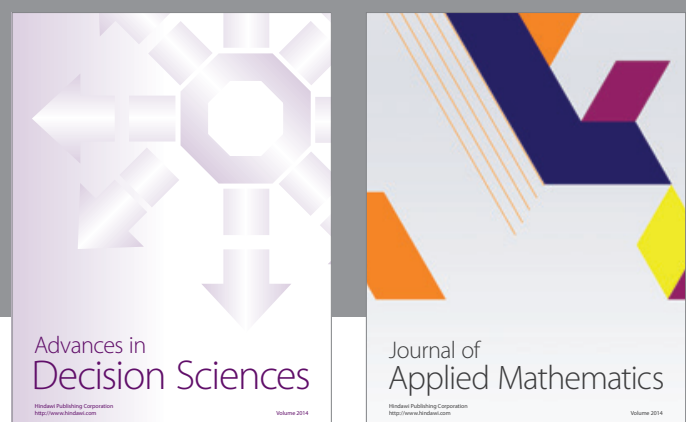

Journal of

Applied Mathematics
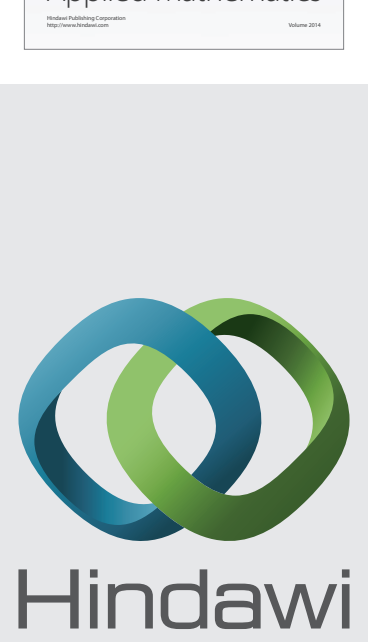

Submit your manuscripts at http://www.hindawi.com
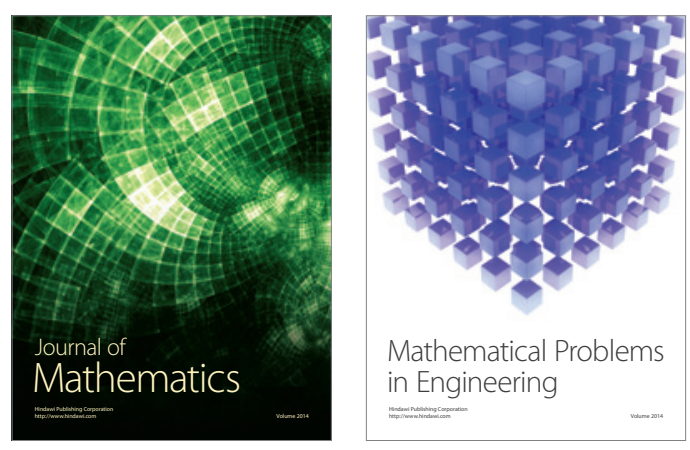

Mathematical Problems in Engineering
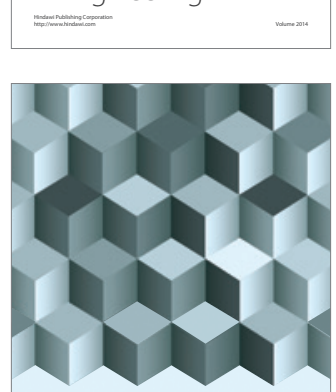

Journal of

Function Spaces
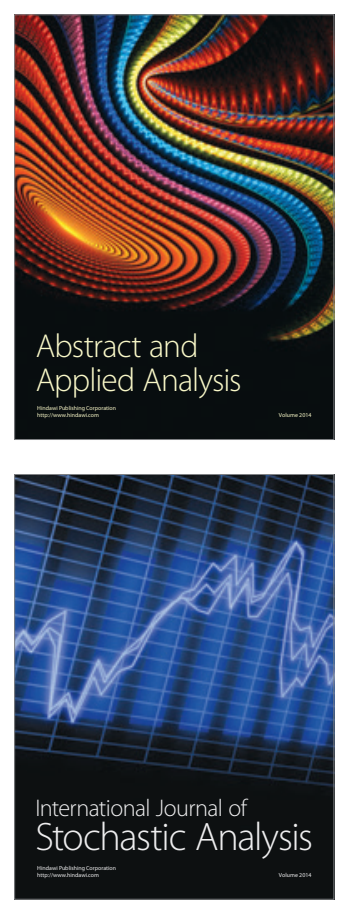

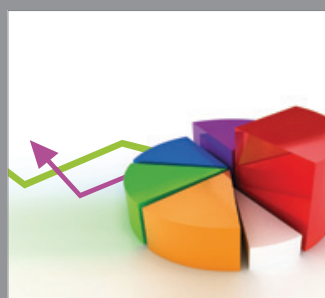

ournal of

Probability and Statistics

Promensencen
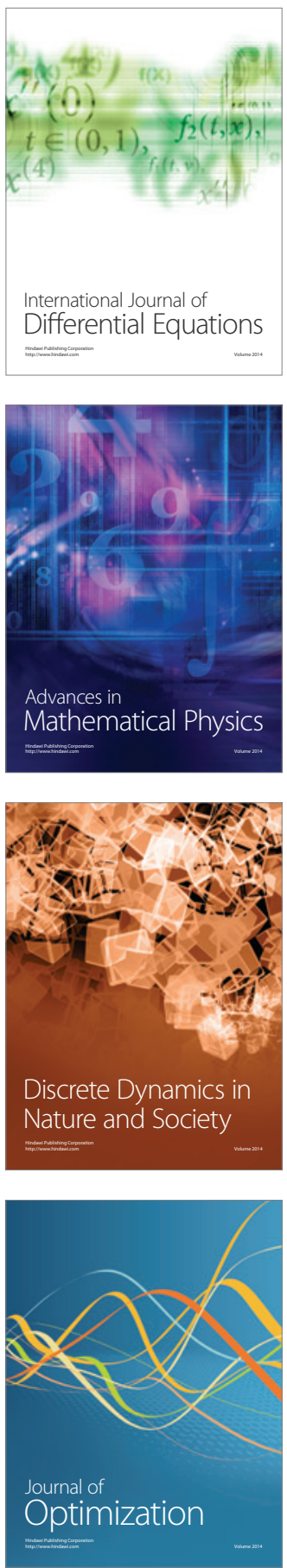